Vol. 39 (1989) [461-469]

\title{
INEQUALITIES AND SEMIGROUPS
}

\author{
W.J. RALPH
}

\begin{abstract}
We define the notion of a convex function between semigroups and show that for such functions one obtains not only Jensen's Inequality but a new and powerful companion inequality. Taken together, these two inequalities can be easily applied to give trivial derivations of many of the classical inequalities as well as many new inequalities.
\end{abstract}

\section{INTRODUCTION}

Substituting "ideas for calculations" has been suggested by N. Bourbaki as one view of mathematics. Our 'idea' here is to prove two theorems in the context of semigroups, namely Theorem $A$ and Theorem $B$ below, which are easy to apply and which virtually eliminate the tedious 'calculations' required to produce so many of the standard inequalities. Furthermore, one comes to understand inequalities more conceptually as simple applications of Theorem A and Theorem B applied to a covex or concave function between two semigroups.

A nice example of this is the function $f:([0, \infty), \cdot)^{n} \rightarrow([0, \infty), \cdot)$ given by $f\left(x_{1}, x_{2}, \cdots, x_{n}\right)=x_{1}+x_{2}+\cdots+x_{n}$. The convexity of this function, in our context, is the Cauchy-Schwarz inequality. Having shown the convexity of $f$, Theorem $\mathbf{A}$ immediately gives Hölder's inequality and Theorem B immediately gives the Power Mean inequality with no further ado.

Our Theorem A is Jensen's inequality in a slightly more general setting that we feel makes it more useful. It is surprising that Theorem $B$ is unknown, since it is so effective in painlessly producing many of the classical inequalities. In a sense, it is a generalisation of the obvious relationship between the slopes of two chords sharing a common end point on the graph of an ordinary convex or concave function.

\section{SEMIgRoups AND CONVEXITY}

The primary goal of this section is to establish the inequalities in Theorem $B, 1.14$. Unlike Jensen's Inequality, the statement of these inequalities requires the language of semigroups. The following definitions proceed to lift the notions of convexity and concavity into that context. We will also establish Jensen's Inequality (Theorem A, 1.7) in that setting.

Received 25 July 1988

Copyright Clearance Centre, Inc. Serial-fee code: 0004-9729/89 \$A2.00+0.00. 
DEFINITION 1.0: As usual, a semigroup is a set $S$ equipped with some associative binary operation. A topological semigroup is a semigroup $S$ for which the binary operation $S \times S \rightarrow S$ is continuous.

Definition 1.1: By the term Real Semigroup we shall always mean either the semigroup $(R,+)$ or $([0, \infty), \cdot)$, where we have used $R$ to denote the real numbers.

Definition 1.2: Let $\Gamma$ denote the set $(0, \infty) \subseteq R$ with the usual operations of addition and multiplication and the usual topology.

Our theorems will be in terms of the action of $\Gamma$ on some semigroup. This action is exactly like the action of the reals on a real vector space with the obvious modifications as given in the following

DEFinition 1.3: We will say that $\Gamma$ acts on a semigroup $S$ if there is a map $\eta: \Gamma \times S \rightarrow S$ such that for any $\alpha, \beta \in \Gamma$ and $s, t \in S$ the following hold, where $\alpha * s \equiv \eta(\alpha, s)$
(i) $1 * s=s$,
(ii) $(\alpha \cdot \beta) * s=\alpha *(\beta * s)$,
(iii) $(\alpha+\beta) * s=(\alpha * s) \cdot(\beta * s)$,
(iv) $\alpha *(s \cdot t)=(\alpha * s) \cdot(\alpha * t)$.

(The reader is cautioned that the operation '.' depends entirely on the context, in particular it may stand for addition of real numbers.)

DEFINITION 1.4: We will say that $\Gamma$ acts continuously on a topological semigroup $S$ if, in addition to the conditions given in (1.3), we also have that for all $s \in S$ the evaluation map $\eta_{\iota}: \Gamma \rightarrow S$, defined by $\eta_{\iota}(\alpha)=\eta(\alpha, s)$, is continuous.

The two primary examples of the type of action specified in (1.3) and (1.4) are the following

$$
\begin{aligned}
& S=((0, \infty), \cdot) \text { with } \alpha * s=s^{\alpha}, \\
& S=(\mathbf{R},+) \text { with } \alpha * s=\alpha \cdot s .
\end{aligned}
$$

From now on, when the action is not explicitly stated, we will assume combinations of the two actions given above. For example, we would assume the action of $\Gamma$ on $([0,1], \cdot) \times([0, \infty),+)$ to be $\alpha *(x, y)=\left(x^{\alpha}, \alpha \cdot y\right)$.

DEFINITION 1.5: Suppose $\Gamma$ acts on a semigroup $S$ as in (1.3). We say a subset $E \subseteq S$ is $\Gamma$-convex if, whenever $\lambda_{i} \in \Gamma$ and $e_{i} \in \Gamma$ and $e_{i} \in E$ with $\sum_{i=1}^{n} \lambda_{i}=1$, we have $\left(\lambda_{1} * e_{1}\right) \cdot\left(\lambda_{2} * e_{2}\right) \cdots\left(\lambda_{n} * e_{n}\right) \in E$.

There are many variants on the notions of convex and concave functions, most of which agree for continuous functions. Since our functions will always be continuous, we have chosen the condition that is simplest to verify in practice. 
Definition 1.6: Suppose $E \subseteq S$ is convex as in (1.5). Let $R$ be a Real Semigroup and suppose that $f: E \rightarrow R$. We will say that with respect to the action of $\Gamma, f$ is

(i) convex if $f\left((1 / 2) *\left(e_{1} e_{2}\right)\right) \leqslant(1 / 2) *\left(f\left(e_{1}\right) f\left(e_{2}\right)\right)$ for all $e_{1}, e_{2} \in E$;

(ii) concave if $f\left((1 / 2) *\left(e_{1} e_{2}\right)\right) \geqslant(1 / 2) *\left(f\left(e_{1}\right) f\left(e_{2}\right)\right)$ for all $e_{1}, e_{2} \in E$.

Here is our version of Jensen's inequality.

Theorem A 1.7. Suppose that $\Gamma$ acts continuously on a topological semigroup $S$. Let $E \subseteq S$ be convex as in (1.5) and let $R$ be a Real Semigroup. Suppose $x_{i} \in E$, $\lambda_{i} \in \Gamma$ and $\sum_{i=1}^{n} \lambda_{i}=1$.

(i) If $f: E \rightarrow R$ is convex as in (1.6) and continuous then

$$
f\left(\prod_{i=1}^{n} \lambda_{i} * x_{i}\right) \leqslant \prod_{i=1}^{n} \lambda_{i} * f\left(x_{i}\right)
$$

(ii) If $f: E \rightarrow R$ is concave as in (1.6) and continuous then

$$
f\left(\prod_{i=1}^{n} \lambda_{i} * x_{i}\right) \geqslant \prod_{i=1}^{n} \lambda_{i} * f\left(x_{i}\right)
$$

Proof: The proof for the case where all the $\lambda_{i}$ are rational is just the usual proof of Jensen's inequality with slight notational changes to allow for the action of $\Gamma$, plus the observation that the 'usual' action of $\Gamma$ on a Real Semigroup preserves the natural ordering. See the proof given in Olkin [7] as a model.

To see that the result holds for all $\lambda_{i} \in \mathbf{R}$ notice, by our assumptions on the continuity of the action of $\Gamma$ on $S$, that for fixed $\left(x_{1}, x_{2}, \cdots, x_{n}\right) \in E^{n}$ the expressions on either side of the inequality sign in $f\left(\prod_{i=1}^{n} \lambda_{i} * x_{i}\right) \leqslant \prod_{i=1}^{n} \lambda_{i} * f\left(x_{i}\right)$ can be regarded as continuous functions of $\left(\lambda_{1}, \lambda_{2}, \cdots, \lambda_{n}\right) \in \Gamma^{n}$. Since the inequality holds on a dense subset of $\Gamma^{\boldsymbol{n}}$ it must hold everywhere.

The rest of this section is devoted to proving Theorem $B$, our main theorem.

Definition 1.8: Suppose $\Gamma$ acts on a semigroup $S$. For $A \subseteq S$ and $\lambda \in \Gamma$, we define

$$
A^{(\lambda)}=\left\{\left(\lambda_{1} * a_{1}\right) \cdot\left(\lambda_{2} * a_{2}\right) \cdots\left(\lambda_{n} * a_{n}\right) \mid a_{i} \in A, \lambda_{i} \in \Gamma, \sum_{i=1}^{n} \lambda_{i}=\lambda \& n \text { arbitrary }\right\}
$$

DEFINITION 1.9: Suppose $\Gamma$ acts on a semigroups $S$ and $E \subseteq S$ is some subset. For any $\lambda \in \Gamma$, we define $\lambda * E=(\lambda * e \mid e \in E)$. 
LEMMA 1.10. If $E \subseteq S$ is $\Gamma$ convex as in (1.5), then $\lambda * E=E^{(\lambda)}$.

Proof: Clearly $\lambda * E \subseteq E^{(\lambda)}$. Now let $\prod_{i=1}^{n} \lambda_{i} * e_{i} \in E^{(\lambda)}$, where $\sum_{i=1}^{n} \lambda_{i}=\lambda$ and $e_{i} \in E$. Then $\prod_{i=1}^{n} \lambda_{i} * e_{i}=\lambda *\left(\prod_{i=1}^{n}\left(\lambda_{i} / \lambda\right) * e_{i}\right) \in \lambda * E$, since $\sum_{i=1}^{n}\left(\lambda_{i} / \lambda\right)=1$ and $E$ is convex.

The following easy lemma is left to the reader.

LEMMA 1.11. Suppose that $\Gamma$ acts on a semigroup $S$ and that $S$ contains an identity element. Then the identity element is fixed under the action of $\Gamma$.

LEMMA 1.12. Suppose $\Gamma$ acts on $S$ and that $E$ is any subset of $S$. Suppose that $S$ contains an identity element $1 \in E$. Then $E^{(\lambda)} \subseteq E^{(\omega)}$ for $\lambda<\omega$.

Proof: Consider $\prod_{i=1}^{n} \lambda_{i} * e_{i} \in E^{(\lambda)}$, where $\sum_{i=1}^{n} \lambda_{i}=\lambda$ and $e_{i} \in E$. We have $\prod_{i=1}^{n} \lambda_{i} * e_{i}=\left(\prod_{i=1}^{n} \lambda_{i} * e_{i}\right) \cdot((\omega-\lambda) * 1)$, since $(\omega-\lambda) * 1=1$, and this is in $E^{(\omega)}$, since $e_{i}, 1 \in E$ and $(\omega-\lambda)+\sum_{i=1}^{N} \lambda_{i}=\omega$.

LEMMA 1.13. Suppose that $\Gamma$ acts continuously on a topological semigroup $S$. Let $E \subseteq S$ be convex and let $R$ be a Real Semigroup with $g: E \rightarrow R$ and $g$ continuous. If $x \in E^{(\lambda)}$ and

(i) g is convex then

$$
\inf \left\{\prod_{i=1}^{n} \lambda_{i} * g\left(e_{i}\right) \mid \prod_{i=1}^{n} \lambda_{i} * e_{i}=x \& \sum_{i=1}^{n} \lambda_{i}=\lambda, e_{i} \in E\right\}=\lambda * g\left(\frac{1}{\lambda} * x\right)
$$

(ii) $g$ is concave then

$$
\sup \left\{\prod_{i=1}^{n} \lambda_{i} * g(e) \mid \prod_{i=1}^{n} \lambda_{i} * e_{i}=x \& \sum_{i=1}^{n} \lambda_{i}=\lambda, e_{i} \in E\right\}=\lambda * g\left(\frac{1}{\lambda} * x\right)
$$


Proof: We will prove (i) only, the other case being similar. We have

$$
\begin{aligned}
& \inf \left\{\prod_{i=1}^{n} \lambda_{i} * g\left(e_{i}\right) \mid \prod_{i=1}^{n} \lambda_{i} * e_{i}=x \& \sum_{i=1}^{n} \lambda_{i}=\lambda, e_{i} \in E\right\} \\
& =\inf \left\{\lambda *\left(\prod_{i=1}^{n}\left(\lambda_{i} / \lambda\right) * g\left(e_{i}\right)\right) \mid \prod_{i=1}^{n}\left(\lambda_{i} / \lambda\right) * e_{i}=(1 / \lambda) * x \& \sum_{i=1}^{n} \lambda_{i}=\lambda, e_{i} \in E\right\} \\
& \geqslant \inf \left\{\lambda * g\left(\prod_{i=1}^{n}\left(\lambda_{i} / \lambda\right) * e_{i}\right) \mid \prod_{i=1}^{n}\left(\lambda_{i} / \lambda\right) * e_{i}\right. \\
& \left.\left.=(1 / \lambda) * x \& \sum_{i=1}^{n} \lambda_{i}=\lambda, e_{i} \in E\right\} \quad \text { by } 1.7 \text { since } g \text { is convex }\right) \\
& =\lambda * g((1 / \lambda) * x) .
\end{aligned}
$$

Since this last number is achieved by $\prod_{i=1}^{n} \lambda_{i} * g\left(e_{i}\right)$, when $e_{i}=(1 / \lambda) * x$ and $\lambda_{i}=\lambda / n$, the result follows.

We are now equipped to prove our main theorem.

Theorem B 1.14. Suppose that $\Gamma$ acts continuously on a topological semigroup $S$. Let $E \subseteq S$ with $E$ convex as in (1.5) and suppose that $S$ has an identity element $1 \in E$. Let $R$ be a Real Semigroup as in (1.1). Suppose that $f: E \rightarrow R$ is continuous and that $f(1)$ is invertible in $R$ with inverse $\Delta$. Then

(i) if $f$ is convex as in (1.6) then

$$
\omega *(\Delta \cdot f((1 / \omega) * x)) \leqslant \lambda *(\Delta \cdot f((1 / \lambda) * x))
$$

for $0<\lambda \leqslant w$ and $x \in \lambda * E$.

(ii) if $f$ is concave as in (1.6) then

$$
\omega *(\Delta \cdot f((1 / \omega) * x)) \geqslant \lambda *(\Delta \cdot f((1 / \lambda) * x))
$$

for $0<\lambda \leqslant \omega$ and $x \in \lambda * E$.

Proof: We will prove part (i) and leave part (ii) to the reader. Define $g: E \rightarrow R$ by $g(x)=\Delta \cdot f(x) \cdot g$ is clearly convex. Now let $\Gamma$ act on $S \times R$ by acting on each factor separately.

Define $A \subseteq S \times R$ by $A=((e, g(e)) \mid e \in E)$. Note that $A$ contains the identity element of $S \times R$ namely (1,g(1)). Apply (1.12) to see that $A^{(\lambda)} \subseteq A^{(\omega)}$ for $0<\lambda \leqslant \omega$. The projection of $A^{(\lambda)}$ onto the first factor is $E^{(\lambda)}$, which equals $\lambda * E$ by (1.10). Fix 
some particular $x \in \lambda * E$. Clearly, for this $x,\left\{y \mid(x, y) \in A^{(\lambda)}\right\} \subseteq\left\{y \mid(x, y) \in A^{(\omega)}\right\}$ so

$$
\inf \left\{y \mid(x, y) \in A^{(\omega)}\right\} \leqslant \inf \left\{y \mid(x, y) \in A^{(\lambda)}\right\} .
$$

Now

$$
\inf \left\{y \mid(x, y) \in A^{(\omega)}\right\}=\inf \left\{\prod_{i=1}^{n} \omega_{i} * g\left(e_{i}\right) \mid \prod_{i=1}^{n} \omega_{i} * e_{i}=x, \sum_{i=1}^{n} \omega_{i}=\omega, e_{i} \in E\right\}
$$

which equals $\omega * g((1 / \omega) * x)$ by (1.13). Similarly $\inf \left\{x \mid(x, y) \in A^{(\lambda)}\right\}=\lambda *$ $g((1 / \lambda) * x)$ and the result follows.

\section{EXAMPLES OF INEQUALITIES THAT FOLLOW FROM THEOREM A AND THEOREM B}

The reader should compare our treatment of the classical inequalities with that given by Hardy [1] or Roberts [8], say, in order to appreciate the extent to which we have eliminated the many ad hoc arguments that normally appear in this theory.

\section{Example 2.1. Hölder's inequality and the power mean inequality.}

Fix $\left(\alpha_{1}, \alpha_{2}, \cdots, \alpha_{n}\right) \in[0, \infty)^{n}$, where not all of the $\alpha_{i}$ are zero, and consider the function $f:([0, \infty), \cdot)^{n} \rightarrow([0, \infty), \cdot)$ given by $f\left(x_{1}, x_{2}, \cdots, x_{n}\right)=\alpha_{1}$. $x_{1}+\alpha_{2} \cdot x_{2}+\cdots+\alpha_{n} \cdot x_{n}$. We claim that $f$ is $\Gamma$ convex. We have to verify that $f((1 / 2) *(x y)) \leqslant(1 / 2) *(f(x) \cdot f(y))$ for all $x, y \in[0, \infty)^{n}$, where $x=$ $\left(x_{1}, x_{2}, \cdots, x_{n}\right)$ and $y=\left(y_{1}, y_{2}, \cdots, y_{n}\right)$. So, in this context, we must show that $f\left(\sqrt{x_{1} \cdot y_{1}}, \sqrt{x_{2} \cdot y_{2}}, \cdots, \sqrt{x_{n} \cdot y_{n}}\right)$ $\leqslant \sqrt{f\left(x_{1}, x_{2}, \cdots, x_{n}\right) \cdot f\left(y_{1}, y_{2}, \cdots, y_{n}\right)}$ or explicitly that

$$
\begin{aligned}
& \alpha_{1} \cdot \sqrt{x_{1} \cdot y_{1}}+\alpha_{2} \cdot \sqrt{x_{2} \cdot y_{2}}+\cdots+\alpha_{n} \cdot \sqrt{x_{n} \cdot y_{n}} \\
& \quad \leqslant \sqrt{\left(\alpha_{1} \cdot x_{1}+\alpha_{2} \cdot x_{2}+\cdots+\alpha_{n} \cdot x_{n}\right) \cdot\left(\alpha_{1} \cdot y_{1}+\alpha_{2} \cdot y_{2}+\cdots+\alpha_{n} \cdot y_{n}\right)},
\end{aligned}
$$

which of course is a form of the Cauchy-Schwarz inequality. We can now write down immediately the inequalities guaranteed by Theorems A and B. Theorem A says that $f\left(\prod_{i=1}^{n} \lambda_{i} * x_{i}\right) \leqslant \prod_{i=1}^{n} \lambda_{i} * f\left(x_{i}\right)$. If we set $x_{i}=\left(x_{i 1}, x_{i 2}, \cdots, x_{i n}\right)$ in this expression we get

$$
\sum_{j=1}^{n}\left(\alpha_{i} \cdot \prod_{i=1}^{n} x_{i j}^{\lambda_{i}}\right) \leqslant \prod_{i=1}^{n}\left(\sum_{j=1}^{n} \alpha_{j} \cdot x_{i j}\right)^{\lambda_{i}}
$$

whenever $\sum_{j=1}^{n} \lambda_{i}=1$, which is Hölder's inequality. 
Now consider Theorem $B$. The identity element of $([0, \infty), \cdot)^{n}$ is $(1,1, \ldots, 1)$ and $\Delta=1 /(f(1,1, \cdots, 1))=1 /\left(\alpha_{1}+\alpha_{2}+\cdots+\alpha_{n}\right)$. So we can write down immediately that

$$
\left(\frac{\alpha_{1} \cdot x_{1}^{1 / \omega}+\alpha_{2} \cdot x_{2}^{1 / \omega}+\cdots+\alpha_{n} \cdot x_{n}^{1 / \omega}}{\alpha_{1}+\alpha_{2}+\cdots+\alpha_{n}}\right)^{\omega} \leqslant\left(\frac{\alpha_{1} \cdot x_{1}^{1 / \lambda}+\alpha_{2} \cdot x_{2}^{1 / \lambda}+\cdots+\alpha_{n} \cdot x_{n}^{1 / \lambda}}{\alpha_{1}+\alpha_{2}+\cdots+\alpha_{n}}\right)^{\lambda}
$$

whenever $0<\lambda \leqslant \omega$, which is the well-known Power Mean inequality.

The integral forms of Hölder's Inequality and the Power Mean inequality can be obtained just as easily in this manner.

\section{Example 2.2. A.M. - G.M. inequality .}

The function $f:([0, \infty), \cdot) \rightarrow(\mathbf{R},+)$ given by $f(x)=x$ is convex. To see this, we have only to verify that $f(1 / 2 *(x \cdot y)) \leqslant 1 / 2 *(f(x) \cdot f(y))$ for all $x, y \in[0, \infty)$. So in this context we must show that $f(\sqrt{x \cdot y}) \leqslant(f(x)+f(y)) / 2$, or explicitly that $\sqrt{x \cdot y} \leqslant(x+y) / 2$.

Theorem A gives $x_{1}^{\lambda_{1}} \cdot x_{2}^{\lambda_{2}} \cdots x_{n}^{\lambda_{n}} \leqslant \lambda_{1} \cdot x_{1}+\lambda_{2} \cdot x_{2}+\cdots+\lambda_{n} \cdot x_{n}$, whenever $\sum_{i=1}^{n} \lambda_{i}=1$ and $x_{i} \geqslant 0$.

To apply Theorem $B$, we substitute $\Delta=-f(1)=-1$ into $\omega *(\Delta \cdot f((1 / \omega) * x)) \leqslant$ $\lambda *(\Delta \cdot f(1 / \lambda) * x)$ and obtain that $\omega \cdot\left(x^{1 / \omega}-1\right) \leqslant \lambda \cdot\left(x^{1 / \lambda}-1\right)$, where $\lambda \leqslant \omega$ and $x \in \lambda *[0, \infty)=[0, \infty)$.

EXAMPLE 2.3: Theorem $B$ applied to the $\Gamma$ concave function $f:([-1, \infty),+) \rightarrow$ $([0, \infty), \cdot)$ defined by $f(x)=1+x$, gives

$$
\left(1+\frac{x}{\lambda}\right)^{\lambda} \leqslant\left(1+\frac{x}{\omega}\right)^{\omega} \text {, whenever } 0<\lambda \leqslant \omega \text { and } x \in \lambda *[-1, \infty)=[-\lambda, \infty) .
$$

EXAMPLE 2.4: The function $f:([0, \infty),+) \rightarrow([0, \infty), \cdot)$ given by $f(r)=$ $\Gamma(r+1)=\int_{0}^{\infty} x^{r} e^{-x} d x$ is ' $\Gamma$ ' convex. Since $f(0)=1$, Theorem B gives $f(x / \omega)^{\omega} \leqslant$ $f(x / \lambda)^{\lambda}$ for $0<\lambda \leqslant \omega$ and $x \in[0, \infty)$. Now let $m$ and $n$ be integers with $0<m \leqslant n$ and substitute $\lambda=1 / n, \omega=1 / m$ and $x=1$ to obtain that

$$
(m !)^{1 / m} \leqslant(n !)^{1 / n} \text { for } 0<m \leqslant n .
$$

We leave it to the reader to write down the result of applying Theorem $A$.

EXAMPLE 2.5: Levine [5] has shown that the following functions (in our terminology ) are $\Gamma$ concave

$$
\begin{gathered}
f:[0, \pi] \subseteq([0, \infty), \cdot) \rightarrow([0, \infty), \cdot) \text { given by } f(x)=\sin x \\
f:[0, \infty) \subseteq([0, \infty), \cdot) \rightarrow([0, \infty), \cdot) \text { given by } f(x)=\log (1+x) \\
f:[0, \infty) \subseteq([0, \infty), \cdot) \rightarrow([0, \infty), \cdot) \text { given by } f(x) 1-\exp (-x)
\end{gathered}
$$


It is also shown in Levine [5] that the following functions are $\Gamma$ convex

$$
\begin{gathered}
f:[0, \infty) \subseteq([0, \infty), \cdot) \rightarrow([0, \infty), \cdot) \text { given by } f(x)=(\exp x)-1 \\
f:[0,1) \subseteq([0, \infty), \cdot) \rightarrow([0, \infty), \cdot) \text { given by } f(x)=-\log (1-x) .
\end{gathered}
$$

We leave it to the reader to write down the inequalities that follow from Theorems $\mathrm{A}$ and $\mathrm{B}$. the inequality obtained by applying Theorem $\mathrm{A}$ to $\sin x$ is given by Lemine [5].

EXAMPLE 2.6: The function $f:([0,1),+) \rightarrow([1, \infty), \cdot)$ given by $f(x)=$ $(1+x) /(1-x)$ is $\Gamma$ convex on $[0,1)$. Theorem $\mathrm{A}$ applied to $f$ gives an inequality due to Klamkin [3]. Theorem $B$ is left to the reader.

To finish, we give some new inequalities.

EXAMPLE 2.7: The function $f:(((x, y) \mid 0 \leqslant x \leqslant y), \cdot) \rightarrow(\mathbf{R},+)$, given by $f(x, y)$ $=\sqrt{(y+x) / 2}+\sqrt{(y-x) / 2}$, is $\Gamma$ convex. Unfortunately, the proof of this is too long to include here.

Theorem A now gives

$$
\begin{aligned}
& \sqrt{\frac{y_{1}^{\lambda_{1}} \cdot y_{2}^{\lambda_{2}} \cdots y_{n}^{\lambda_{n}}+x_{1}^{\lambda_{1}} \cdot x_{2}^{\lambda_{2}} \cdots x_{n}^{\lambda_{n}}}{2}}+\sqrt{\frac{y_{1}^{\lambda_{1}} \cdot y_{2}^{\lambda_{2}} \cdots y_{n}^{\lambda_{n}}-x_{1}^{\lambda_{1}} \cdot x_{2}^{\lambda_{2}} \cdots x_{n}^{\lambda_{n}}}{2}} \\
& \leqslant \lambda_{1} \cdot\left(\sqrt{\frac{y_{1}+x_{1}}{2}}+\sqrt{\frac{y_{1}-x_{1}}{2}}\right)+\lambda_{2} \cdot\left(\sqrt{\frac{y_{2}+x_{2}}{2}}+\sqrt{\frac{y_{2}-x_{2}}{2}}\right)+\cdots \\
& \quad+\lambda_{n} \cdot\left(\sqrt{\frac{y_{n}+x_{n}}{2}}+\sqrt{\frac{y_{n}-x_{n}}{2}}\right),
\end{aligned}
$$

where $0 \leqslant x_{i} \leqslant y_{i}, \lambda_{i}>0$ and $\sum_{i=1}^{n} \lambda_{i}=1$.

Theorem $B$ gives

$$
\begin{aligned}
& \omega \cdot\left(\sqrt{\frac{y^{1 / \omega}+x^{1 / \omega}}{2}}+\sqrt{\frac{y^{1 / \omega}-x^{1 / \omega}}{2}}-1\right) \\
& \quad \leqslant \lambda \cdot\left(\sqrt{\frac{y^{1 / \lambda}+x^{1 / \lambda}}{2}}+\sqrt{\frac{y^{1 / \lambda}-x^{1 / \lambda}}{2}}-1\right)
\end{aligned}
$$

where $0<\lambda \leqslant \omega$ and $(x, y) \in \lambda *\{(x, y) \mid 0 \leqslant x \leqslant y\}=\{(x, y) \mid 0 \leqslant x \leqslant y\}$.

\section{REFERENCES}

[1] G.H. Hardy, J.E. Littlewood and G. Polya, Inequalities (Cambridge Univ. Press, London and New York, 1st ed. 1934, 2nd ed. 1952). 
[2] N.D, Kazarinoff, Analytic inequalities (Holt, Rinehart and Winston, New York, 1961).

[3] M.S. Klamkin, 'Extensions of the Weeirstrass product inequalities II', Amer. Math. Monthly 82 (1975), 741-742.

[4] M.S. Klamkin and D.J. Newman, 'Extensions of the Weierstrass product inequalities', Math. Mag 43 (1970), 137-141.

[5] E. Levine and N. Schaumberger, 'Comparability of $\sin \sqrt{x y}$ and $\sqrt{\sin x \cdot \sin y}$, College Math. J. 17 (1986), 362-363.

[6] D.S. Mitrinovic, Elementary inequalities (Noordhall, Groningen, 1964).

[7] I. Olkin and A.W. Marshall, Inequalities: Theory of majorization and its applications (Academic Press, New York, 1979).

[8] A.W. Roberts and D.E. Varberg, Convex functions (Academic Press, New York, 1973).

Department of Mathematics

Brock University

St. Catharines, Ontario, L2S 3A1

Canada 